Supplement of Atmos. Chem. Phys. Discuss., 14, 22217-22243, 2014

http://www.atmos-chem-phys-discuss.net/14/22217/2014/

doi:10.5194/acpd-14-22217-2014-supplement

(C) Author(s) 2014. CC Attribution 3.0 License.

(c) (i)

Supplement of

\title{
Iodine oxide in the global marine boundary layer
}

C. Prados-Roman et al.

Correspondence to: A. Saiz-Lopez (a.saiz@csic.es) 


\section{Methods}

\subsection{Measurements of IO during Malaspina 2010}

\subsubsection{Spectral retrieval of IO}

The analysis of IO on the 417-439 nm spectral region was performed as described in the study of Mahajan et al. (2012), with the updated $\mathrm{H}_{2} \mathrm{O}$ water cross-section (Rothman et al, 2013). Glyoxal (CHOCHO) was not included in the spectral fit of IO since CHOCHO was under the instrumental detection limit (Mahajan et al., 2014). Aiming at increasing the signal-to-noise ratio, spectra were accumulated during $1 \mathrm{~h}$, resulting in a residual root-mean-square (RMS) of 2-6 $\times 10^{-4}$ and a mean $2 \sigma$ detection limit of $1.2-3.5 \times 10^{13}$ molecules $\mathrm{cm}^{-2}$ for IO. An example of a typical spectral fit of IO is shown in Fig. 3a. Similar to previous studies (Sinreich et al., 2010; Mahajan et al., 2012; Gómez Martín et al., 2013a; Großmann et al., 2013), the following filters were included in the DOAS data analysis for quality assurance:

- The data collected at $\mathrm{SZA}>60^{\circ}$ were excluded in the analysis (minimizing possible contribution of stratospheric trace gases).

- Saturated spectra that occurred around noon at some locations were also excluded before the DOAS analysis.

- The upper limit allowed for the RMS of the DOAS fit was of $10^{-3}$.

- A wind direction filter was applied, preventing data in which the ship's exhaust plume crossed the MAX-DOAS field-of-view.

- A cloud filter was also introduced based on in-situ measured solar radiation and radiances measured at the edges of the chip of the CCD camera of the MAX-DOAS (400 and $480 \mathrm{~nm}$ in this case). With this cloud filter a threshold for the cloud-free scenario was set, assuring therefore consistency in the scattering conditions between all the data measured under clear sky. An example of this filter is shown in Fig. S1. 
- In order to assure measurements representative of open marine conditions, data collected while the vessel was in a harbour or close to it were excluded from the data set.

Data above/below these quality filters were considered statistically relevant/irrelevant as presented in Fig. 3b. Only statistically relevant data were used for the retrieval of IO vmr.

\subsubsection{Retrieval of IO mixing ratios}

Following the approach of previous studies (e.g., Sinreich et al., 2010; Mahajan et al., 2012; Gómez Martín et al., 2013a), the IO mixing ratios along the ship track were inferred from the measured IO dSCD applying the $\mathrm{O}_{4}$ method (Wagner et al., 2004; Frieß et al., 2006) after validating that method with the IO mixing ratios inferred by the inversion method, i.e., by means of a RTM (NIMO, Hay et al. (2012)) combined with the optimal estimation approach (Rodgers, 2000). Since the $\mathrm{O}_{4}$ method and the inversion method have been both widely used for retrieving mixing ratios in the MBL, no details on the methods are provided herein. For the rationale and further details behind either of these methods, please refer to the above mentioned references.

Briefly, in the $\mathrm{O}_{4}$ method the atmospheric scattering conditions are characterised and, consequently, the light path needed to infer mixing ratios from dSCD. In order to retrieve the IO mixing ratios by applying this method, the $\mathrm{O}_{4} \mathrm{dSCD}$ along the Malaspina's ship track were measured in the 338-370 $\mathrm{nm}$ spectral region. For this vmr retrieval exercise, only the dSCD for the $2^{\circ}$ elevation angle $(\alpha)$ were used and a scaling factor was applied to transfer the scattering conditions inferred in the UV to the spectral range of IO (further details in Mahajan et al., 2012; Gómez Martín et al., 2013a). Sensitivity studies performed with the NIMO RTM (Hay et al., 2012) with data collected along the cruise indicated a last scattering altitude between 250-1300 $\mathrm{m}$ for $\alpha=2^{\circ}$, with $600 \mathrm{~m}$ as the mean last scattering altitude (LSA). Hence the IO mixing ratios obtained through the $\mathrm{O}_{4}$ method should be regarded as an averaged value within the first $600 \mathrm{~m}$ of the troposphere. Similarly, the degrees of freedom (0.6-0.8) and averaging kernels obtained after applying the inversion method indicated that the retrieved IO vmr vertical profile was smoothed in the first $600-800 \mathrm{~m}$, therefore not gaining any additional information by applying the costly inversion method. Nevertheless, for validation purposes, vertical profiles of IO vmr were inferred by the inversion approach for several days during 
1 the different legs of the circumnavigation. For this inversion approach, in order to 2 characterise the scattering properties of the atmosphere, a vertical profile of the aerosol 3 extinction coefficient (EC) was previously inferred through forward modelling $\mathrm{O}_{4} \mathrm{dSCD}$ 4 (Wagner et al., 2004; Frieß et al., 2006). An example of the derived aerosol EC is shown in 5 Fig. S2a, with a vertical profile decreasing rapidly with height. Note that the MAX-DOAS instrument during Malaspina 2010 was placed at 10 m.a.s.1 and no measurements were performed at negative elevation angles. Hence the EC inferred below that altitude should be regarded with caution. Nevertheless in all cases only an aerosol EC vertical profile type as Fig. S2 -with relatively high values up to $10 \mathrm{~m}$ altitude and virtually zero above $30 \mathrm{~m}$ - could reproduce measured $\mathrm{O}_{4} \mathrm{dSCD}$ for all elevation angles. This sort of EC profile was included in the model in order to invert the IO vmr vertical profiles. Based on previous works (e.g. Mahajan et al., 2012) the surface albedo used in the RTM was of 7\% and the aerosols were assumed to be of marine origin (asymmetry parameter 0.75 and single scattering albedo 0.97). The vertical grid for the vmr inversion exercise was of $100 \mathrm{~m}$. Examples of the inverted IO vmr through this inversion approach are shown in Fig. S2b.

Figure S3 provides the comparison of the IO vmr obtained from the $\mathrm{O}_{4}$ method and from the inversion approach considering the modelled LSA $=600 \mathrm{~m}$ altitude. As shown in the figure, the IO vmr inferred with both methods showed a good agreement with more than $99 \%$ confidence, although values gained after the $\mathrm{O}_{4}$ method overestimated the IO vmr by about $15 \%$. That factor was hence applied to the $\mathrm{O}_{4}$ method-derived IO vmr reported in this study.

\section{$21 \quad$ Error analysis}

22 Herein we discuss the error characterisation of the IO vmr inferred by both methods. In Fig. S3, the error bars of the IO vmr inferred through the inversion method derive from the optimal estimation equations (Rodgers, 2000), being the measurement error (18\%) the dominant error source. The error related to the IO vmr derived from the $O_{4}$ method is rather complex to estimate given the different assumptions related to the method itself such as a similar shape of the vertical profile of $\mathrm{IO}$ and $\mathrm{O}_{4}$, or the presence of a homogenous IO layer until the last scattering altitude (e.g., Wagner et al., 2004; Mahajan et al., 2012; Gómez Martín et al., 2013a). The IO vmr values reported in the main text were obtained through this $\mathrm{O}_{4}$ method and the error bars provided (Fig. 5 and Fig. S3) derived from the IO and $\mathrm{O}_{4}$ dSCD 31 measurement errors and from forward RTM modelling sensitivity studies for different aerosol loads and IO profile shapes. Overall, we estimated a 30\% uncertainty for the retrieved IO vmr reported in this work, albeit probably underestimated. 


\subsection{Ancillary data during Malaspina 2010: measurements vs. model}

Since the newly included formulation of ISG in the CAM-Chem model depends on $\mathrm{O}_{3}$, wind speed and sea surface temperature (Carpenter et al., 2013; MacDonald et al., 2014), aiming at testing the model performance, we compared observations of those parameters observed during Malaspina 2010 with the modelled ones. Figure S4 shows the comparison of the modelled daily averages (9:00-17:00 LT) of the three parameters computed for each Malaspina's time and geolocation, with daytime values observed during the cruise in the period where IO was also observed. As shown in the figure, the model climatology reproduces well the experimental measurements, with relative deviations smaller than $15 \%$. From the three parameters, SST showed the best representation with $\mathrm{R}^{2}=0.91$ and $99.9 \%$ confidence. Note that wind speeds measured along the Malaspina's transect were above the threshold of $3 \mathrm{~m} \mathrm{~s}^{-1}$ set by MacDonald et al. (2014) for the validity of the ISG parameterisation. Further discussions on the intrinsic limitations and uncertainties of the parameterisation of ISG flux are addressed elsewhere (Carpenter et al., 2013; MacDonald et al., 2014; Prados-Roman et al., 2014).

\subsection{Modelled $\mathrm{O}_{3}$ in the global marine environment}

For completeness of results shown in the main text, Fig. S5 provides the globally modelled $\mathrm{O}_{3}$ mixing ratios. Modelled values and spatial distribution agree with observations (e.g., Myhre et al., 2013).

\section{References unique to this SI}

Frieß, U., Monks, P. S., Remedios, J. J., Rozanov, A., Sinreich, R., Wagner, T., and Platt, U.: MAX-DOAS $\mathrm{O}_{4}$ measurements: A new technique to derive information on atmospheric aerosols: 2. Modeling studies, J. Geophys. Res.,: Atmospheres, 111, D14203, 10.1029/2005JD006618, 2006.

Hay, T. D., Bodeker, G. E., Kreher, K., Schofield, R., Liley, J. B., Scherer, M., and McDonald, A. J.: The NIMO Monte Carlo model for box-air-mass factor and radiance calculations, Journal of Quantitative Spectroscopy and Radiative Transfer, 113, 721-738, http://dx.doi.org/10.1016/j.jqsrt.2012.02.005, 2012. 
1 Rodgers, C. D.: Inverse methods for atmospheric sounding: Theory and Practice, Series on

2 Atmospheric, Oceanic and Planetary Physics-Vol. 2, Singapore, World Scientific, 2000.

3 Rothman, L. S. et al.: The HITRAN 2012 molecular spectroscopic database. JQSTR 130, 4$450,2013$.

5 Sinreich, R., Coburn, S., Dix, B., and Volkamer, R.: Ship-based detection of glyoxal over the 6 remote tropical Pacific Ocean, Atmos. Chem. Phys., 10, 11359-11371, doi:10.5194/acp-107 11359-2010, 2010.

8 


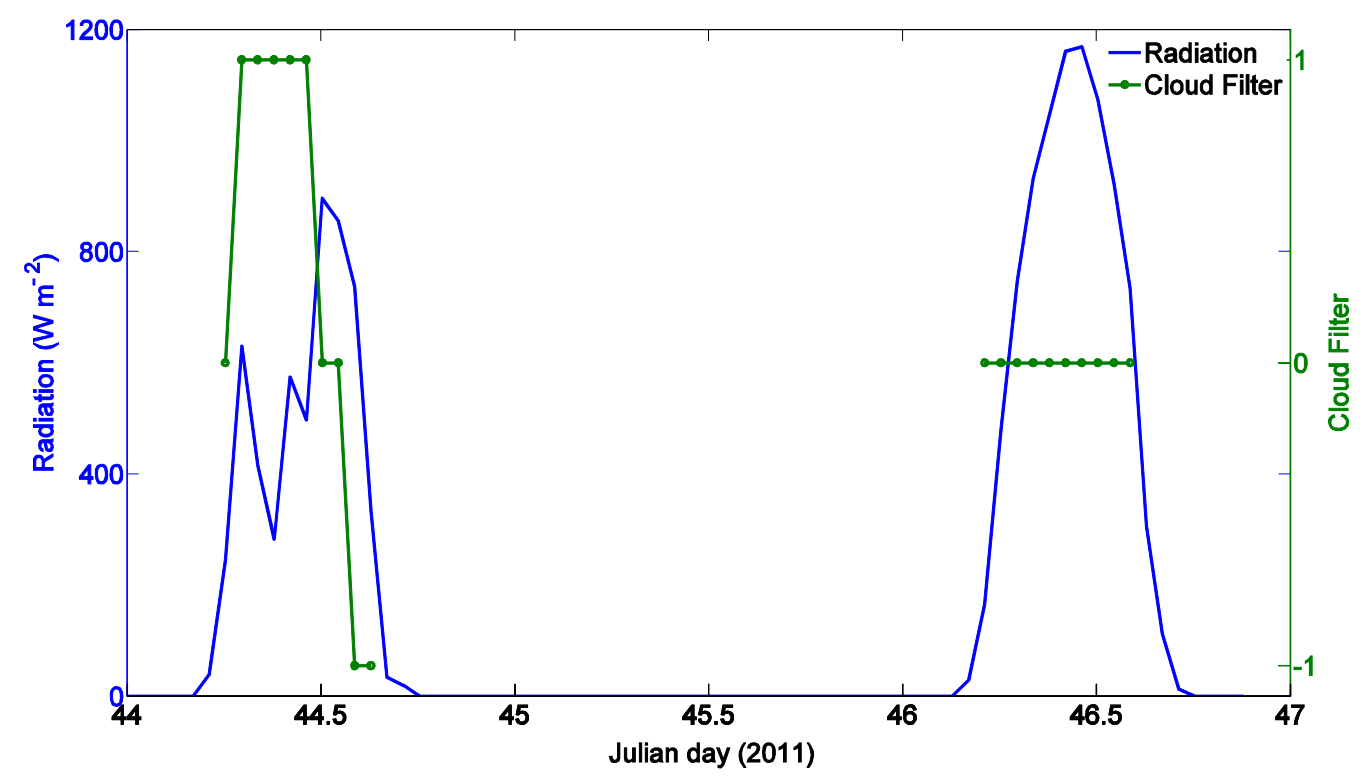

3 Figure S1. Example of the cloud filter applied in the Malaspina dataset. The left axis indicates

4 the in-situ measured radiation (in blue). The right axis corresponds to the cloud filter (in 5 green) and indicates cloud-free conditions for 0 , cloudy conditions for 1 and missing data for 61 (neglected therefore).

7

8 


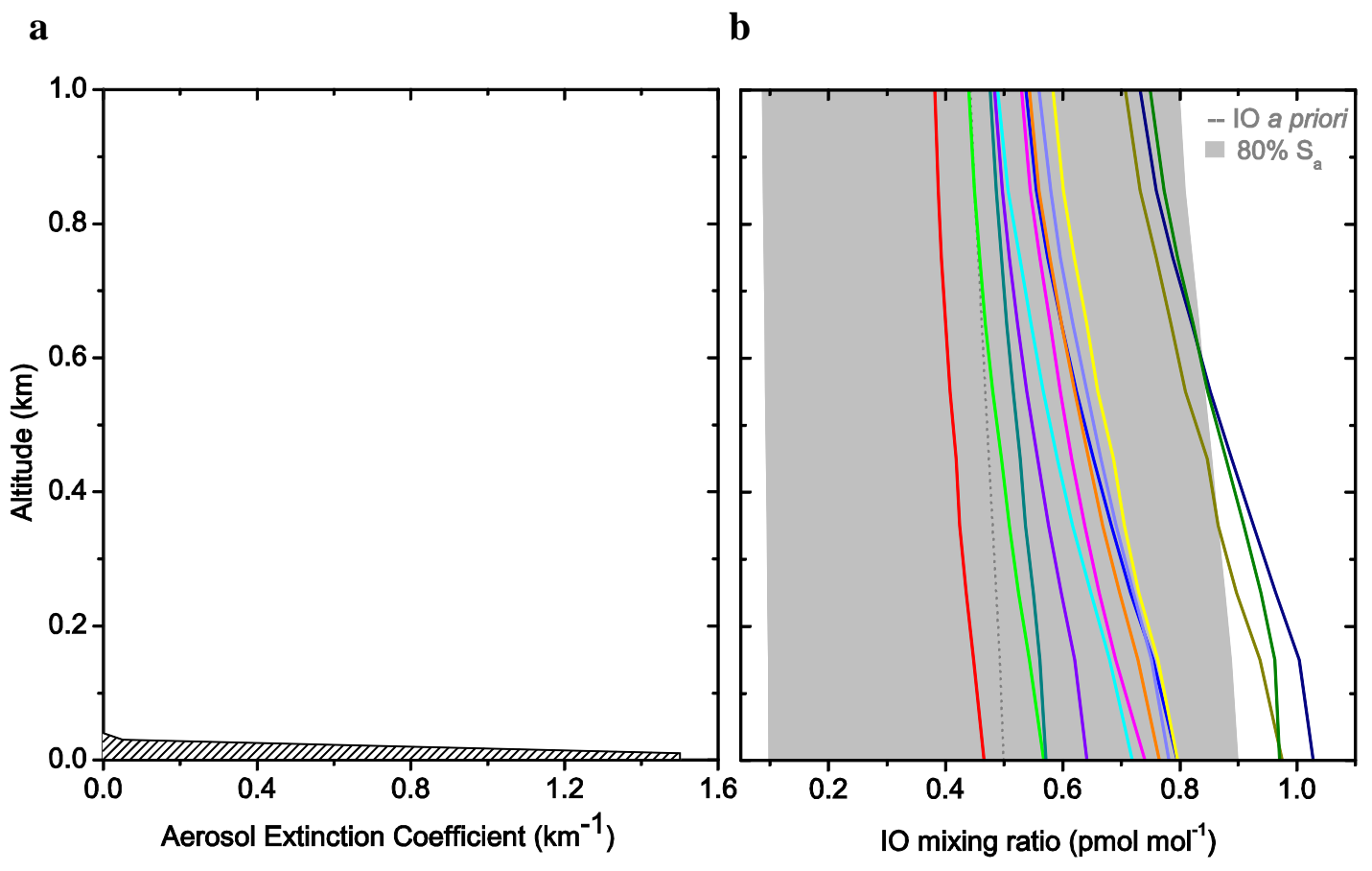

2 Figure S2. Inversion method for retrieving vertical profiles of IO mixing ratios. a. In 3 consistency with previous works (Mahajan et al., 2012; Gómez Martín et al., 2013a), this 4 panels shows the typical aerosol extinction coefficient considered in the RTM (inferred after $5 \mathrm{O}_{4} \mathrm{dSCD}$ forward modelling) for the inversion of IO vmr vertical profiles. b. Profiles of IO 6 vmr inverted in the first kilometre of the MBL for several days during Malaspina 2010. The 7 dashed gray line is the a priori IO used, with its covariance indicated by the gray shadow. The 8 solid lines show the IO vmr profiles inverted (100 m vertical grid). 


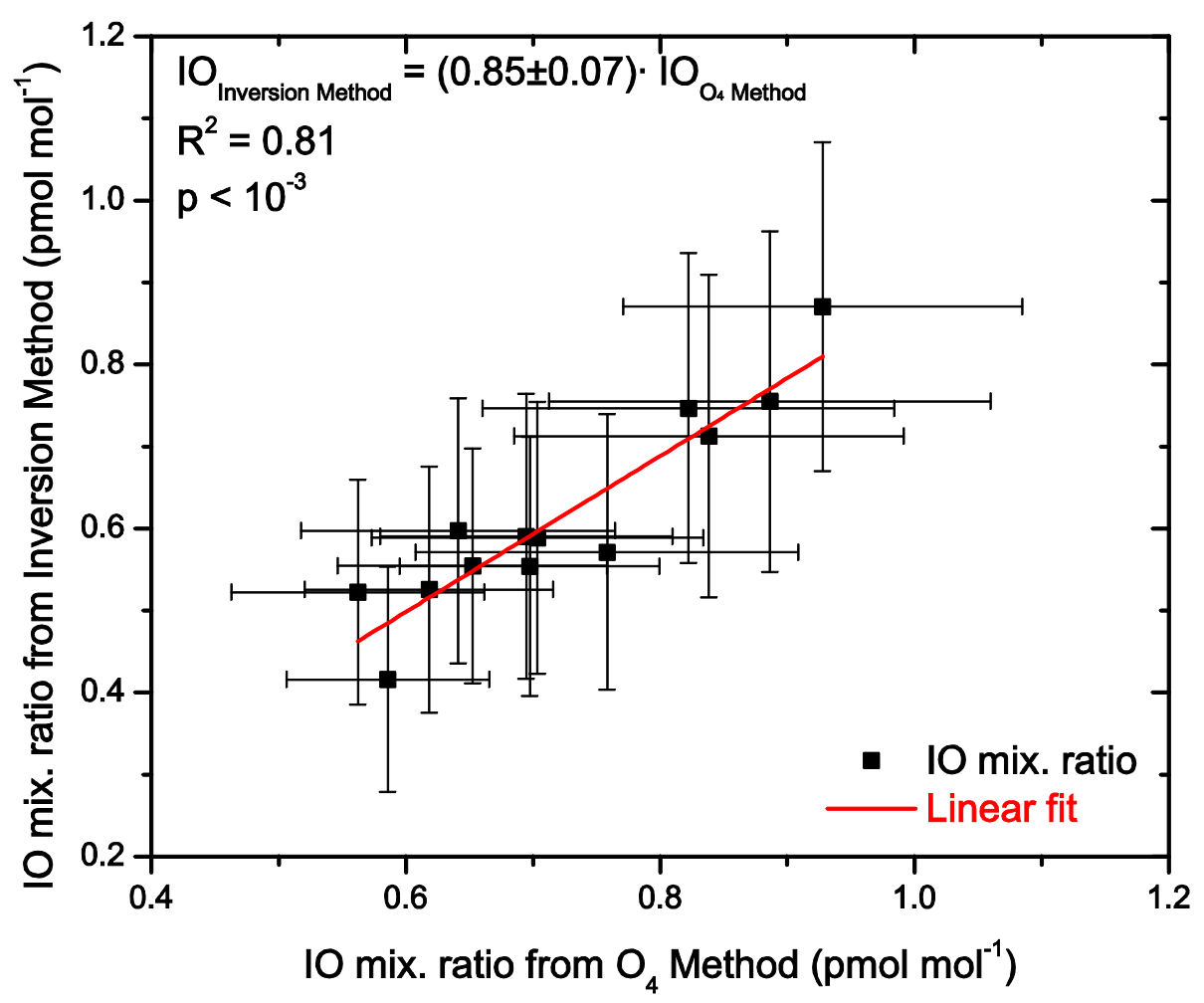

1

2 Figure S3. Comparison of the IO mixing ratios along the Malaspina's transect derived from 3 the $\mathrm{O}_{4}$ method and from the inversion approach. The linear fit (dashed gray line) of the 4 inverted mixing ratios (black squares) indicates a high correlation between the two retrieval 5 methods, with a confidence level higher than $99 \%$.

6 

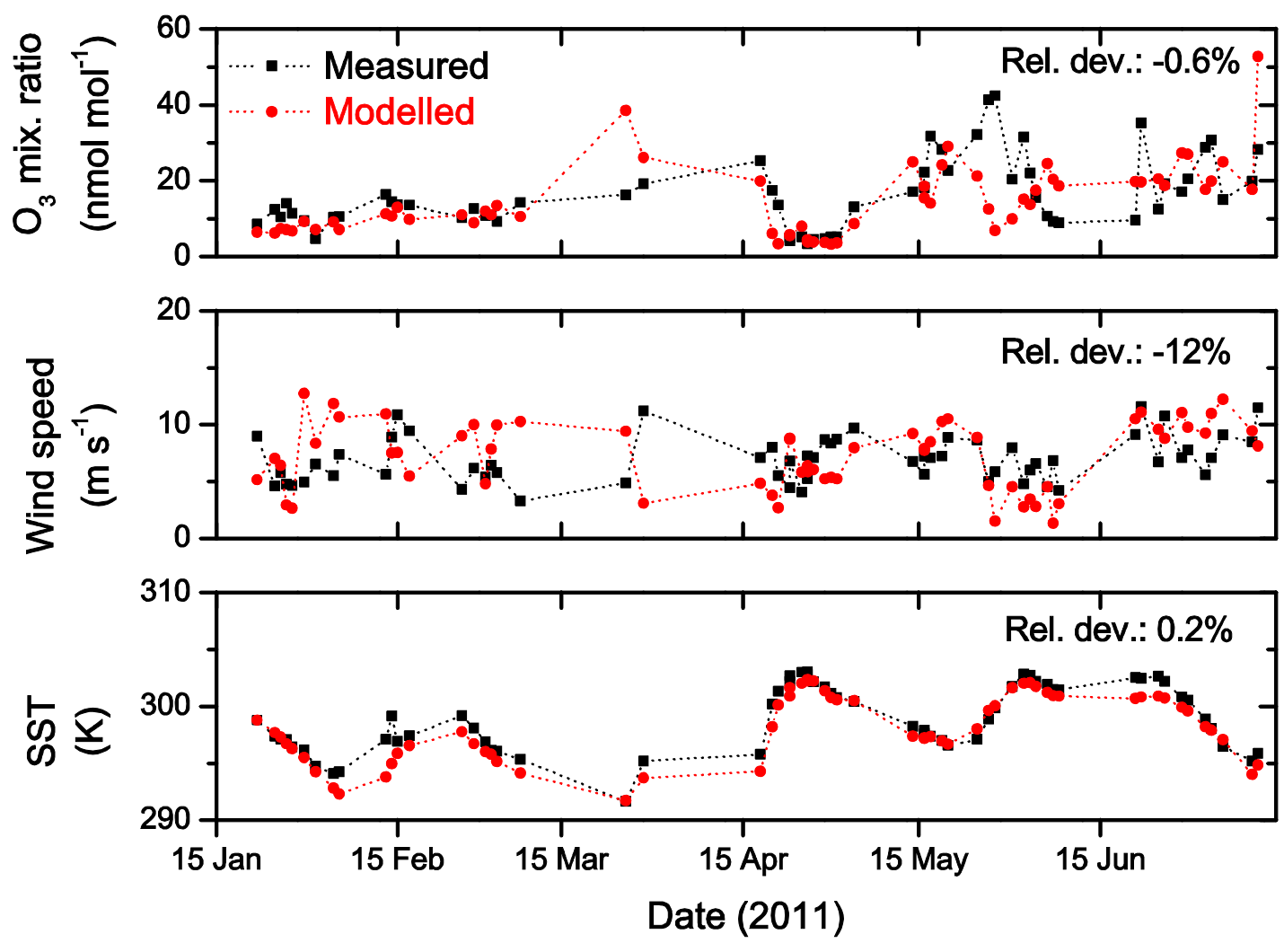

2 Figure S4. Measured and modelled daytime values of surface $\mathrm{O}_{3}$ mixing ratios (upper panel), 3 wind speed (middle panel) and sea surface temperature (SST, lower panel) during the 4 Malaspina campaign. The relative deviation of each modelled values (i.e., difference of the 5 measured and modelled values relative to measured ones) is also provided as "Rel. dev.". 


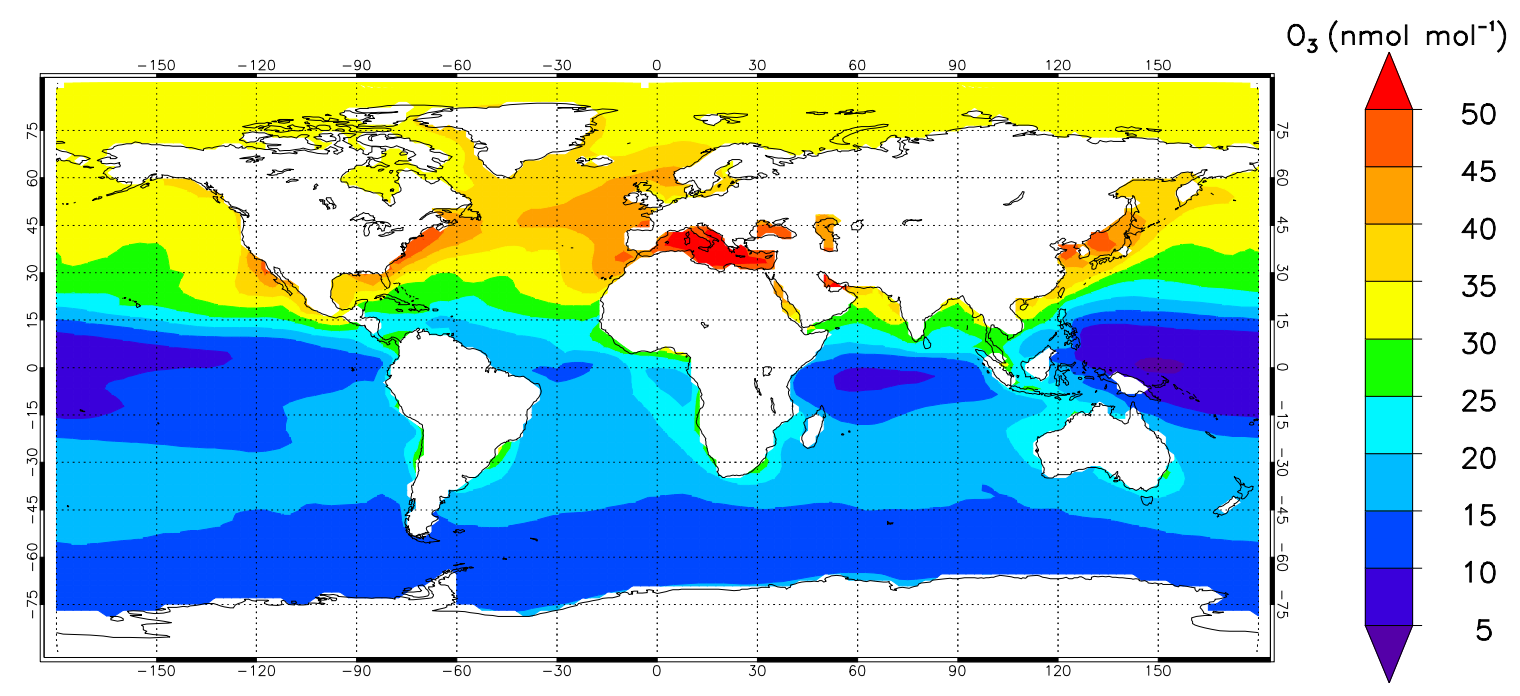

1

2 Figure S5. Modelled annual averaged surface ozone mixing ratios in the marine environment. 